\title{
Acoustic waves in a stratified atmosphere
}

\section{Temperature inhomogeneities}

\author{
G. Bodo ${ }^{1}$, W. Kalkofen ${ }^{2}$, S. Massaglia ${ }^{3}$, and P. Rossi ${ }^{1}$ \\ 1 Osservatorio Astronomico di Torino, Strada dell'Osservatorio 20, 10025 Pino Torinese, Italy \\ e-mail: rossi@to.astro.it \\ 2 Harvard-Smithsonian Center for Astrophysics, Cambridge, Massachusetts 02138, USA \\ e-mail: wolf@cfa.harvard.edu \\ 3 Dipartimento di Fisica Generale dell'Università, Via Pietro Giuria 1, 10125 Torino, Italy \\ e-mail: massaglia@ph.unito.it
}

Received 3 December 1999 / Accepted 15 February 2001

\begin{abstract}
In a gravitationally stratified atmosphere, small temperature variations distort the paths of acoustic waves from the rectilinear paths in an isothermal atmosphere. For temperature increasing upward, low-frequency waves near the acoustic cutoff frequency propagating at a given polar angle are refracted towards the vertical direction (focused) and high-frequency waves, away from the vertical (defocused). Similarly, for temperature increasing towards the axis of a vertical cylinder, low-frequency waves are focused and high-frequency waves are defocused. This effect of temperature inhomogeneities may be important for wave propagation in the chromospheric $K_{2 \mathrm{v}}$ bright point phenomenon.
\end{abstract}

Key words. hydrodynamics - Sun: chromosphere - waves

\section{Introduction}

The usual analytical and numerical treatments of acoustic wave propagation in a gravitationally stratified atmosphere are for plane waves in a one-dimensional medium (Ulmschneider 1974; Fleck \& Schmitz 1991; Carlsson \& Stein 1994; Kalkofen et al. 1994). If the atmosphere is isothermal and semi infinite, a plane wave front of an acoustic wave remains plane, independent of the direction of propagation, and the waves are neither reflected nor refracted. When the atmosphere is thermally inhomogeneous, wave fronts become distorted, and the effect of the inhomogeneity depends on frequency since acoustic waves in a gravitationally stratified atmosphere are dispersive, i.e., their group and phase velocities depend on frequency.

The solution of the three-dimensional wave equation for an isothermal atmosphere stratified in plane-parallel layers has been discussed by Bodo et al. (2000; hereafter BKMR). We will now investigate the effects of a temperature variation on the propagation characteristics of acoustic waves, assuming that the solution of the threedimensional wave equation for the isothermal medium holds locally so that the dispersion relation is unchanged, except that the sound speed and the acoustic cutoff frequency now depend on position in the atmosphere.

Send offprint requests to: G. Bodo, e-mail: bodo@to.astro.it
We consider two special cases of temperature variation: 1) the temperature increases monotonically upward, starting at $z=0$, and 2) the temperature drops from its maximal value on the axis of a vertical cylinder down to a constant value far from the axis.

The plan of the paper is the following: In Sect. 2 we write the basic equations for wave propagation; in Sect. 3 we apply the model to a stratified, non-isothermal atmosphere and discuss the results, and in Sect. 4 we give a simple physical interpretation of the behavior of wave paths and draw conclusions.

\section{Basic equations}

We consider a small temperature variation of an isothermal, stratified medium, assuming that the scale length of the temperature variation is much larger than typical wavelengths (i.e. we perform a local analysis). Following Whitham (1974), we define a phase $\theta(\boldsymbol{x}, t)$, where $\boldsymbol{x}=$ $\left(x_{1}, x_{2}, x_{3}\right)$, and where frequency $\omega$ and vector wavenumber $\boldsymbol{k}$ are given by

$\omega=-\frac{\partial \theta}{\partial t}, \quad k_{i}=\frac{\partial \theta}{\partial x_{i}}$ 
Frequency and wavenumber are related by the local dispersion relation

$\omega=W(\boldsymbol{k}, \boldsymbol{x})$.

Eliminating $\theta$ from Eq. (1) we have

$\frac{\partial k_{i}}{\partial t}+\frac{\partial \omega}{\partial x_{i}}=0, \quad \frac{\partial k_{i}}{\partial x_{j}}-\frac{\partial k_{j}}{\partial x_{i}}=0$

Introducing $\omega=W(\boldsymbol{k}, \boldsymbol{x})$ into the first of Eqs. (2) we obtain

$\frac{\partial k_{i}}{\partial t}+\frac{\partial W}{\partial k_{j}} \frac{\partial k_{j}}{\partial x_{i}}=-\frac{\partial W}{\partial x_{i}}$

and using the second of Eqs. (2),

$\frac{\partial k_{i}}{\partial t}+C_{j} \frac{\partial k_{i}}{\partial x_{j}}=-\frac{\partial W}{\partial x_{i}}$,

where $\boldsymbol{C}(\boldsymbol{k}, \boldsymbol{x})$ is the group velocity vector defined by

$C_{j}(\boldsymbol{k}, \boldsymbol{x})=\frac{\partial W(\boldsymbol{k}, \boldsymbol{x})}{\partial k_{j}}$.

Equation (3) can now be written as (Whitham 1974, Eq. (11.46))

$\frac{\mathrm{d} k_{i}}{\mathrm{~d} t}=-\frac{\partial W}{\partial x_{i}}, \quad$ where $\frac{\mathrm{d}}{\mathrm{d} t} \equiv \frac{\partial}{\partial t}+C_{j} \frac{\partial}{\partial x_{j}}$

on group lines (or characteristics), which are defined by

$\frac{\mathrm{d} x_{i}}{\mathrm{~d} t}=C_{i}$.

Group lines give the propagation direction of the wave energy. At any position in the medium, the group velocity vector $\boldsymbol{C}$ is tangent to these lines. Note that $\boldsymbol{C}$ in a dispersive medium is a function of wave number $\boldsymbol{k}$ and position $\boldsymbol{x}$. The energy for a given value of $\boldsymbol{k}$ does not in general travel in the direction $\boldsymbol{k} / k$. Only for $(k, \omega) \rightarrow \infty$ do group lines coincide with ray paths, which are along $\boldsymbol{k}$ and perpendicular to wavefronts.

\section{Gravitationally stratified atmosphere}

The dispersion relation for an isothermal atmosphere stratified in the vertical direction, $z=x_{3}$, is (Bray \& Loughhead 1974, BKMR)

$\omega^{2}=\frac{1}{2}\left[\left(k^{2}+1\right) \pm \sqrt{\left(k^{2}+1\right)^{2}-16 \frac{\gamma-1}{\gamma^{2}} k_{\perp}^{2}}\right]$,

where $k^{2}=k_{z}^{2}+k_{\perp}^{2}, \omega$ is in units of the acoustic cutoff frequency, $\omega_{\mathrm{co}}$, and the wavenumber is nondimensionalized with $2 H$, where $H$ is the pressure scale height. The + or sign refers, respectively, to the acoustic and gravity modes. In the following we consider only acoustic modes $(+\operatorname{sign})$.

The relation between the acoustic cutoff frequency and the scale height is

$\omega_{\mathrm{co}}=\frac{a}{2 H}$, where $a$ is the sound speed. The dispersion relation (4) can be written in dimensional form as

$$
\begin{aligned}
\tilde{\omega}^{2} & =\frac{1}{2}\left(\tilde{k}^{2} a^{2}+\omega_{\mathrm{co}}^{2}\right) \\
& +\frac{1}{2} \sqrt{\left(\tilde{k}^{2} a^{2}+\omega_{\mathrm{co}}^{2}\right)^{2}-16 \frac{\gamma-1}{\gamma^{2}} \tilde{k}_{\perp}^{2} a^{2} \omega_{\mathrm{co}}^{2}},
\end{aligned}
$$

where $\tilde{\omega}$ and $\tilde{k}$ are the dimensional forms of $\omega$ and $k$. The dependence on temperature of the various quantities is

$H \propto T, \quad a \propto \sqrt{T}, \quad \omega_{\text {co }} \propto 1 / \sqrt{T}$,

where for a non-isothermal medium $T=T\left(x_{\perp}, z\right)$, with $x_{\perp}=\left(x_{1}, x_{2}\right)$.

Assuming a small temperature variation in the vertical direction and defining $\beta=T / T_{0}$, where $T_{0}$ is the temperature of the isothermal atmosphere, we can write the dispersion relation, nondimensionalized relative to the isothermal atmosphere, as ("local analysis")

$\omega^{2}=\frac{1}{2}\left\{\left[k^{2} \beta\left(x_{\perp}, z\right)+1 / \beta\left(x_{\perp}, z\right)\right]+Y\left(\boldsymbol{k}, x_{\perp}, z\right)\right\}$,

with

$Y\left(\boldsymbol{k}, x_{\perp}, z\right)=\sqrt{\left[k^{2} \beta\left(x_{\perp}, z\right)+1 / \beta\left(x_{\perp}, z\right)\right]^{2}-16 \frac{\gamma-1}{\gamma^{2}} k_{\perp}^{2}}$.

The group velocity components are

$\begin{aligned} C_{z}= & \frac{1}{2} \frac{k_{z}}{\omega} \beta\left(x_{\perp}, z\right) \\ & \times\left\{1+\left[k^{2} \beta\left(x_{\perp}, z\right)+1 / \beta\left(x_{\perp}, z\right)\right] / Y\left(\boldsymbol{k}, x_{\perp}, z\right)\right\},\end{aligned}$

$C_{\perp}=\frac{1}{2} \frac{k_{\perp}}{\omega} \beta\left(x_{\perp}, z\right)\left\{1+\left[k^{2} \beta\left(x_{\perp}, z\right)+1 / \beta\left(x_{\perp}, z\right)\right.\right.$

$\left.\left.-8(\gamma-1) / \gamma^{2} / \beta\left(x_{\perp}, z\right)\right] / Y\left(\boldsymbol{k}, x_{\perp}, z\right)\right\}$.

The spatial derivatives of the frequency are given by

$$
\begin{aligned}
\frac{\partial W}{\partial x_{\perp}, z} & =\frac{1}{4 \omega} \frac{\partial \beta}{\partial x_{\perp}, z}\left[k^{2}-1 / \beta^{2}\left(x_{\perp}, z\right)\right] \\
& \times\left\{1+\left[k^{2} \beta\left(x_{\perp}, z\right)+1 / \beta\left(x_{\perp}, z\right)\right] / Y\left(\boldsymbol{k}, x_{\perp}, z\right)\right\}
\end{aligned}
$$

for the $x_{\perp}$ and $z$ components.

In order to determine the group lines we integrate the equations for the characteristics,

$$
\begin{aligned}
\frac{\mathrm{d} z}{\mathrm{~d} t} & =C_{z}, \\
\frac{\mathrm{d} x_{\perp}}{\mathrm{d} t} & =C_{\perp}, \\
\frac{\mathrm{d} k_{z}}{\mathrm{~d} t} & =-\frac{\partial W}{\partial z}, \\
\frac{\mathrm{d} k_{\perp}}{\mathrm{d} t} & =-\frac{\partial W}{\partial x_{\perp}} .
\end{aligned}
$$

We consider inhomogeneity of the medium for the two special cases of purely vertical $(\beta=\beta(z))$ and purely horizontal $\left(\beta=\beta\left(x_{\perp}\right)\right)$ temperature variations. In the first case we assume a linear spatial dependence, $\beta(z)=1+\beta^{\prime} z$, with $\beta^{\prime}=$ constant, and in the second case, a Gaussian 
G. Bodo et al.: Acoustic waves in stratified atmospheres. III.

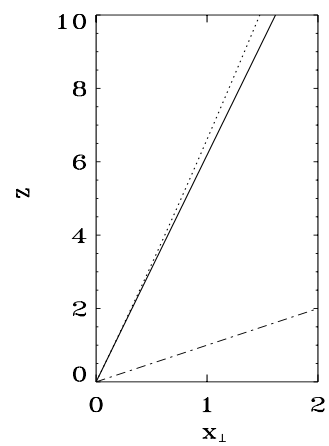

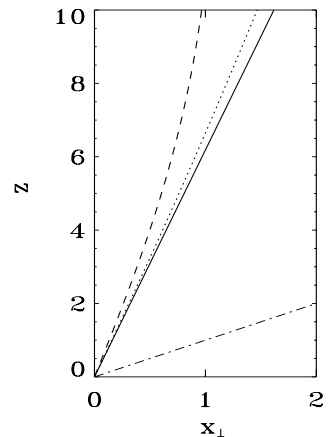

Fig. 1. The group lines for $k=0.5$ and $\psi=45^{\circ}$. Left panel, for vertical temperature variation: isothermal atmosphere (solid line); perturbed atmosphere, with $\beta^{\prime}=0.01$ (dotted line). Right panel, for horizontal temperature variation: $x_{0}=1$, $\epsilon=0.01$ (dotted line) and $\epsilon=0.05$ (dashed line). Dot-dashed lines on both panels indicate the ray paths for an isothermal atmosphere
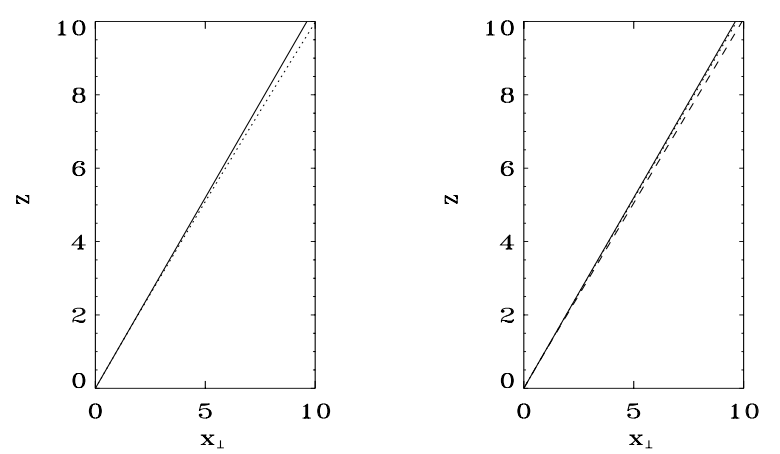

Fig. 2. Same as Fig. 1, but for $k=5$

dependence, $\beta\left(x_{\perp}\right)=1+\epsilon \exp \left[-\left(x_{\perp} / x_{0}\right)^{2}\right]$, where $\epsilon$ and $x_{0}$ are the amplitude and the half width, respectively.

The local analysis, on which our equations are based, breaks down when the wavelength of the acoustic waves, $\lambda=2 \pi / k$, becomes comparable to the characteristic scale, $L$, of the temperature inhomogeneity. This defines a limiting wave number, $k_{\lim }=2 \pi / L$ for the regime of validity of the equations; our results are applicable when $k>k_{\text {lim }}$. For the vertical variation, this limiting wave number is $k_{\lim }=2 \pi \beta^{\prime}$, and for the horizontal variation, $k_{\lim } \approx 5 \epsilon$.

Figure 1 shows the group lines for the wave number $k=0.5$ and initial polar angle of $\psi=45^{\circ}$ between the propagation direction and the $z$ axis, i.e., $k_{z}=k \cos \psi$, $k_{\perp}=k \sin \psi$, and $k_{z}=k_{\perp}$ in the present case. The left panel compares the group line path for a vertical temperature variation, with $\beta^{\prime}=0.01$ (dotted line), and the rectilinear path for an isothermal atmosphere (solid line). The figure shows the focusing of low-frequency waves in which group lines bend towards the $z$ axis. Ray paths for an isothermal atmosphere are also plotted (dot-dashed lines) showing clearly that the wave energy, which is carried along the group lines, is concentrated towards the vertical axis (see BKMR and Kato 1966). Similarly, the right panel shows the focusing of waves for a horizontal temperature variation, with $x_{0}=1$ and the two cases of $\epsilon=0.01$ (dotted line) and $\epsilon=0.05$ (dashed line), again compared
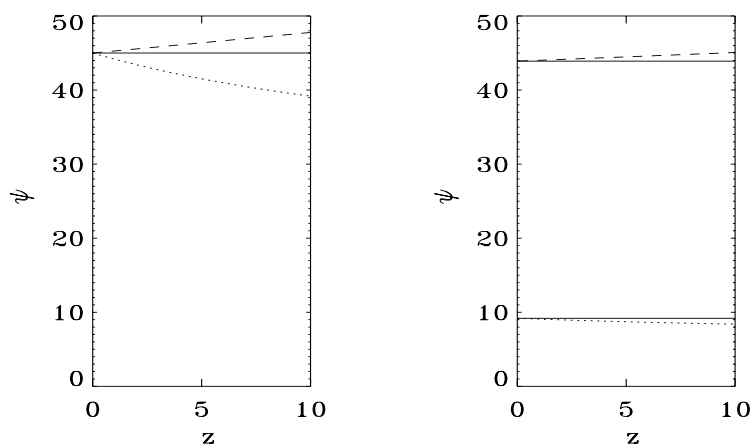

Fig. 3. The polar angle $\psi$ as a function of $z$ for the vertical temperature variation. Left panel: Ray paths for the isothermal atmosphere (solid line) and for $\beta^{\prime}=0.01$ and $k=0.5$ (dotted line) and $k=5$ (dashed line). Right panel: Group lines for the same values of $\beta$ and $k$

to the rectilinear propagation in an isothermal medium (solid line). The opposite case, that of defocusing for highfrequency waves, is shown in Fig. 2 for $k=5$, again compared with the rectilinear propagation (solid line) and for the same parameters as in Fig. 1. Figures 1 and 2 indicate that focusing is more effective than defocusing for both vertical and horizontal temperature variations (note the scaling on the $\left(x_{\perp}, z\right)$ axes in the two figures).

For isothermal models, group lines, defined by Eqs. (5), and ray paths, defined by the direction $\boldsymbol{k} / k$, are straight lines, but with different inclinations with respect to the $z$ axis, except in the limit $(k, \omega) \rightarrow \infty$. But the behavior of ray paths follows that of group lines in focusing and defocusing, as seen in Fig. 3, which shows the polar angle $\psi$ as a function of height $z$ for ray paths (left panel) and group lines (right panel) for $k=0.5$ (dotted lines) and $k=5$ (dashed lines); $\psi$ decreases for $k=0.5$ (focusing) and increases for $k=5$ (defocusing) for both group lines and ray paths. This allows the physical interpretation of the results in terms of ray paths. Note also that the initial inclination of the group lines differs from $\psi=45^{\circ}$ (Fig. 3, right panel), especially for smaller values of $k$.

To understand the nature of the solution of Eqs. (5), note that the wave numbers $k_{z}$ and $k_{\perp}$ are constant along the characteristics when the partial derivatives of the wave frequency $W$ are zero. This occurs for $k=1 / \beta$. Considering the case $k<1 / \beta$, i.e., long wavelengths, for the vertical temperature variation $(\beta=\beta(z))$, we note that the derivative $\partial W / \partial z$ is negative and hence $k_{z}$ grows along the characteristics. Since $k_{\perp}$ is constant $\left(\partial \beta / \partial k_{\perp}=0\right)$, the vertical wave number grows whereas the horizontal wave number does not. Since $\boldsymbol{k} / k$ is the direction of propagation of the wave, i.e., defines the ray path, the rays are bent towards the vertical direction. Thus the waves at long wavelengths are focused by the vertical temperature variation. Similarly, for the horizontal temperature variation $\left(\beta=\beta\left(x_{\perp}\right)\right)$, the partial derivative $\partial \beta / \partial z=0$, hence $\partial W / \partial z=0$ and $\mathrm{d} k_{z} / \mathrm{d} t=0$. Thus $k_{z}$ is constant. But since $\partial \beta / \partial x_{\perp}<0$, the horizontal wave number decreases along the characteristic, resulting again 
in a bending of the rays towards the vertical direction, i.e., focusing of the rays. Defocusing occurs in both types of temperature variation when $k>1 / \beta$, i.e., for short wavelengths.

\section{Discussion and conclusions}

Wave propagation in the real atmosphere is along curved paths. An interesting question to ask is how straight paths are distorted by inhomogeneities in the medium. This can be easily investigated if the departures from idealized conditions are small and the wave amplitude is low. But it must be kept in mind that the interesting effects in the solar chromosphere, for example, are due to large-amplitude waves that form shocks. We suppose that the effect of mild inhomogeneities on the propagation of low-amplitude waves is in the same sense as the effect of strong inhomogeneities on large-amplitude waves, so the investigation of this paper allows us to understand the qualitative behavior of waves in an astrophysically interesting medium like the solar chromosphere.

We ask how propagation along a straight line that represents the outward normal of a wave front is changed in two limiting situations, the temperature varying with height only and the temperature varying in the horizontal direction only.

For the first case we treat an isothermal atmosphere with a temperature variation increasing with height and consider a plane wave front inclined "to the right" relative to the vertical direction. Two special cases are of interest: high-frequency waves and waves with frequency near the acoustic cutoff frequency.

High-frequency waves $(k, \omega \rightarrow \infty)$ are (practically) nondispersive. Their propagation speed is the sound speed, $a$, which depends on temperature as $\sqrt{T}$. Now, the left edge of the wave front is at a higher temperature than the right edge. Points on the left have therefore higher speed and advance with respect to points on the right. The normal of the wave front is bent away from the vertical direction. If we imagine a wave to propagate in a vertical channel, this bending away from the axis of the channel amounts to a defocusing of the wave.

For low-frequency waves, the (local) acoustic cutoff frequency decreases with height because of the increasing temperature, and the phase velocity of waves near the cutoff also decreases with height. The right edge of an inclined plane wave therefore advances relative to the left edge, the wave front is bent towards the vertical and hence towards the axis of a propagation channel. Low-frequency waves therefore are focused.

The second case is that of an atmosphere that is isothermal except for a positive temperature variation that decreases to zero with horizontal distance from a vertical axis. The temperature is highest on the axis. Consequently the sound speed is highest on the axis and the phase velocity is lowest on the axis. Therefore, high-frequency waves, which are practically nondispersive, propagate faster near the axis and hence are bent away

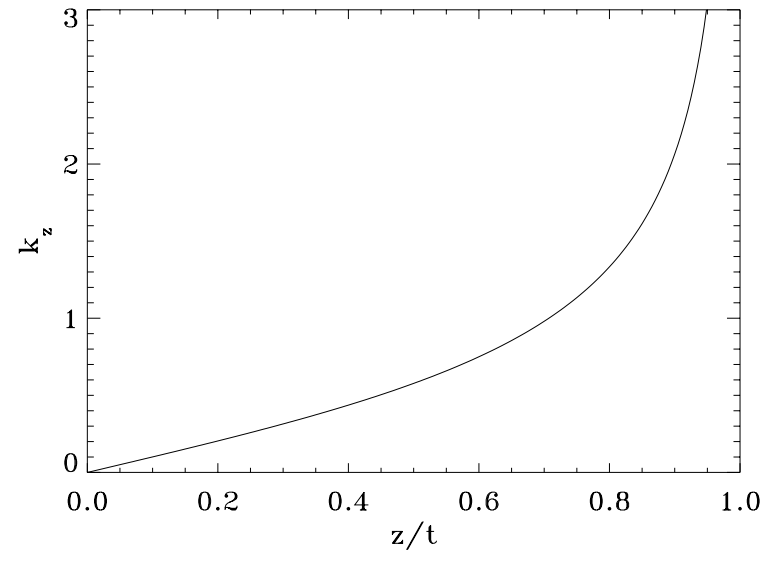

Fig. 4. Vertical distribution of wavenumbers behind the head of the wave front, which is at $z / t=1$ (from BKMR)

from the axis, and low-frequency waves, which are dispersive, have higher phase velocity further from the axis and are bent towards it. Thus, considering wave motion near a hotter propagation channel, high-frequency waves are defocused and low-frequency waves are focused. The cases of temperature increasing with height and increasing towards a vertical axis are thus qualitatively similar.

It is interesting to note that the solar chromosphere has a temperature rising in the outward direction, and magnetic flux tubes in the magnetic network have higher temperature inside than outside. Thus the features of the temperature variations in this paper are qualitatively the same as features of the chromosphere. When one considers impulsively generated acoustic perturbations emanating from a point source and propagating as acoustic waves in an isothermal, stratified medium (see BKMR), one finds that the outermost front carries high-frequency components and expands as a spherical wave at the sound speed, while the wake that follows carries low-frequency components mostly (Fig. 4). The effects described in this paper suggest that the pulse should be defocused and the wake components should be focused towards the vertical direction.

Acknowledgements. This work has been supported by NASA and NSF.

\section{References}

Bodo, G., Kalkofen, W., Massaglia, S., \& Rossi, P. 2000, A\&A, 354, 296 (BKMR)

Bray, R. J., \& Loughhead, R. E. 1974, The Solar Atmosphere (Chapman and Hall, London)

Carlsson, M., \& Stein, R. F. 1997, ApJ, 481, 500

Fleck, B., \& Schmitz, F. 1991, A\&A, 250, 235

Kalkofen, W., Rossi, P., Bodo, G., \& Massaglia, S. 1994, A\&A, 284,976

Kato, S. 1966, ApJ, 144, 326

Ulmschneider, P. 1974, Solar Phys., 39, 327

Whitham, G. B. 1974, Linear and nonlinear waves (John Wiley \& Sons, New York) 